\title{
An Introduction of the New Customer Loyalty Measurement in the Japanese Market
}

\author{
Tatsuya Kimura \\ Waseda University
}

\begin{abstract}
Many companies are using the Net Promoter Score (NPS) proposed by Reichheld (2003) as a replacement for traditional customer satisfaction indices to measure the relationship between customer loyalty and company growth. It is because of its simplicity of structure and ease of use for management; however, there are many criticisms that this scoring concept does not consider the culture and consumer characteristics of the country where it is used. In general, surveys are often affected by response bias, where participant responses may be influenced by various factors unknown to the researchers, one of which is response style. This study aims to examine the adaptability of the NPS in the Japanese market from the perspective of measurement criteria to avoid the current bias, thereby proposing the new concept as an adjustment. Focusing on the response style of Japanese consumers, the author investigates a more appropriate adaptation of the NPS measurement scale based on empirical analysis of customer response data from multiple companies and proposes a new measurement framework in Japan, the Promoter Score Japan (PSJ).
\end{abstract}

Keywords: customer value; loyalty measurement; NPS; PSJ; response bias 